Supporting Information:

\title{
Self-assembled peptide nanostructures targeting death receptor 5 and encapsulating paclitaxel as a multifunctional cancer therapy
}

Tyson J. Moyer, Feng Chen, Daniel J. Toft, Yves Ruff, Vincent L. Cryns, * and Samuel I. Stupp*

\section{Supporting Figures:}

A

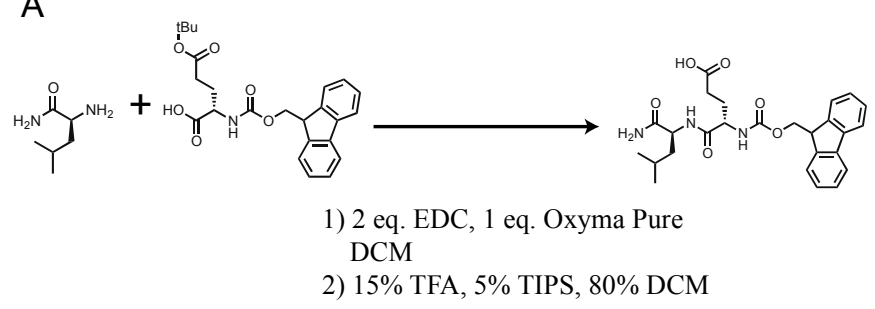

B
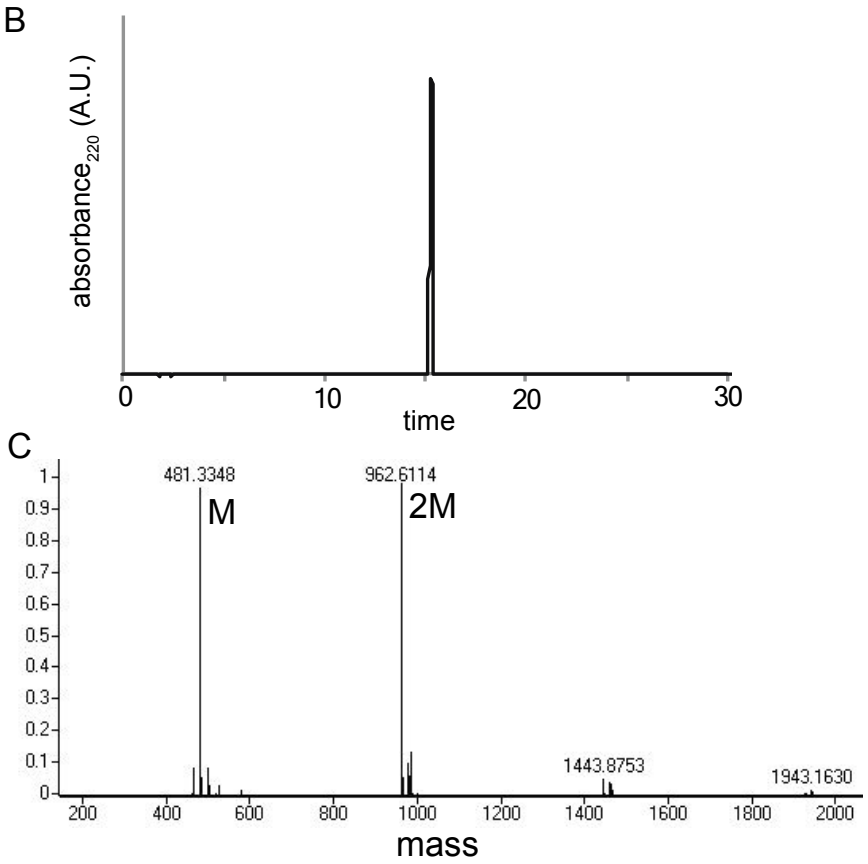

Figure S1: Synthesis and characterization of Fmoc-EL-NH2. (A) Scheme for synthesis of Fmoc-EL$\mathrm{NH}_{2}$ using EDC chemistry to link the two amino acids, followed by deprotection of the tert-butyl group. (B) HPLC of the resulting product shows one major peak. (C) MS of the peak shows the expected mass of the product along with the dimer (962.6). 


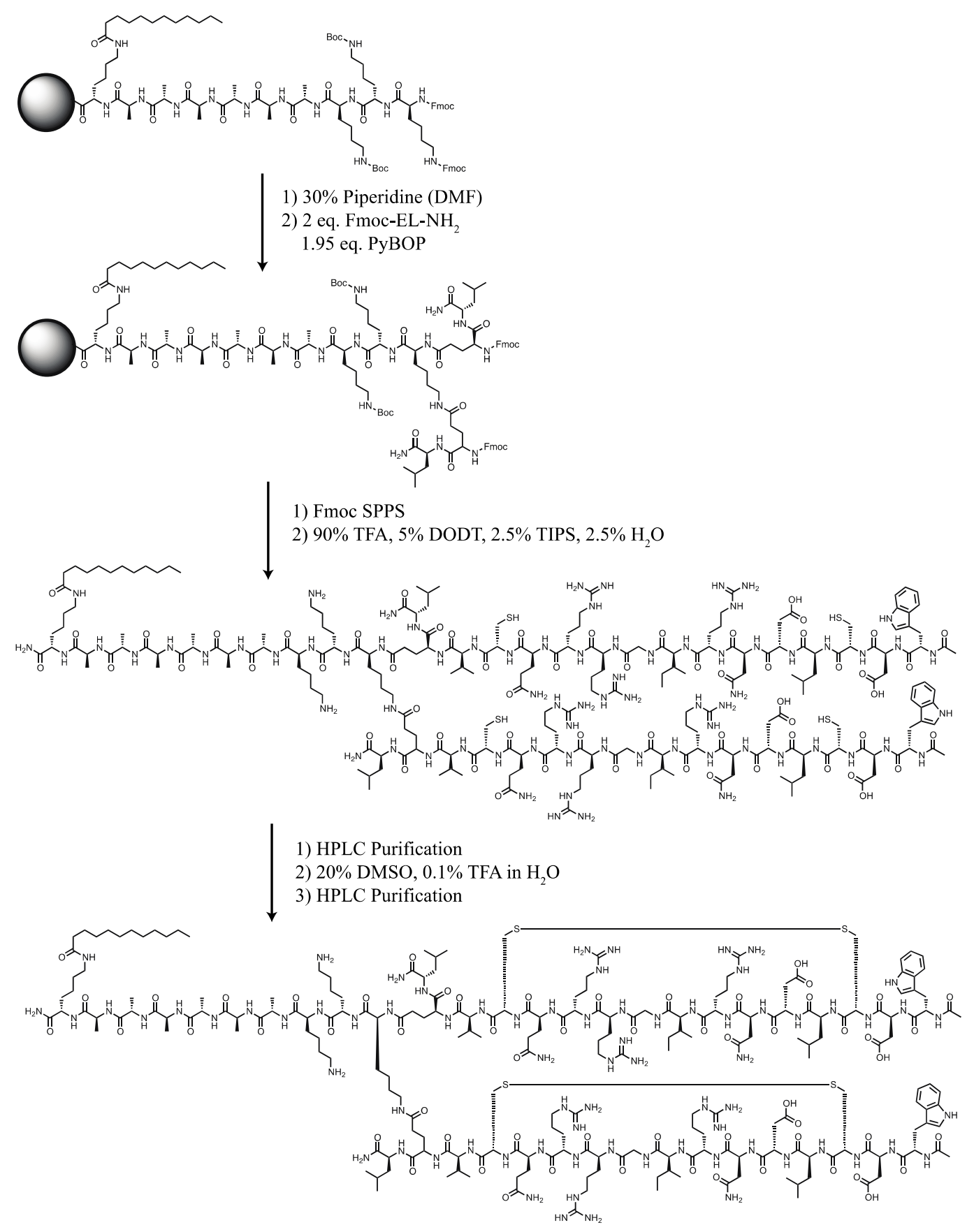

Figure S2: Synthetic scheme for the synthesis of the cyclized PA 1 by SPPS. A Fmoc-Lys(fmoc)$\mathrm{COOH}$ is used to form the dimeric structure, and Fmoc-EL-NH $\mathrm{N}_{2}$ from supporting figure 1 is used to preserve the exact binding sequence. Cyclization is performed off resin in the presence of DMSO as the oxidant. 


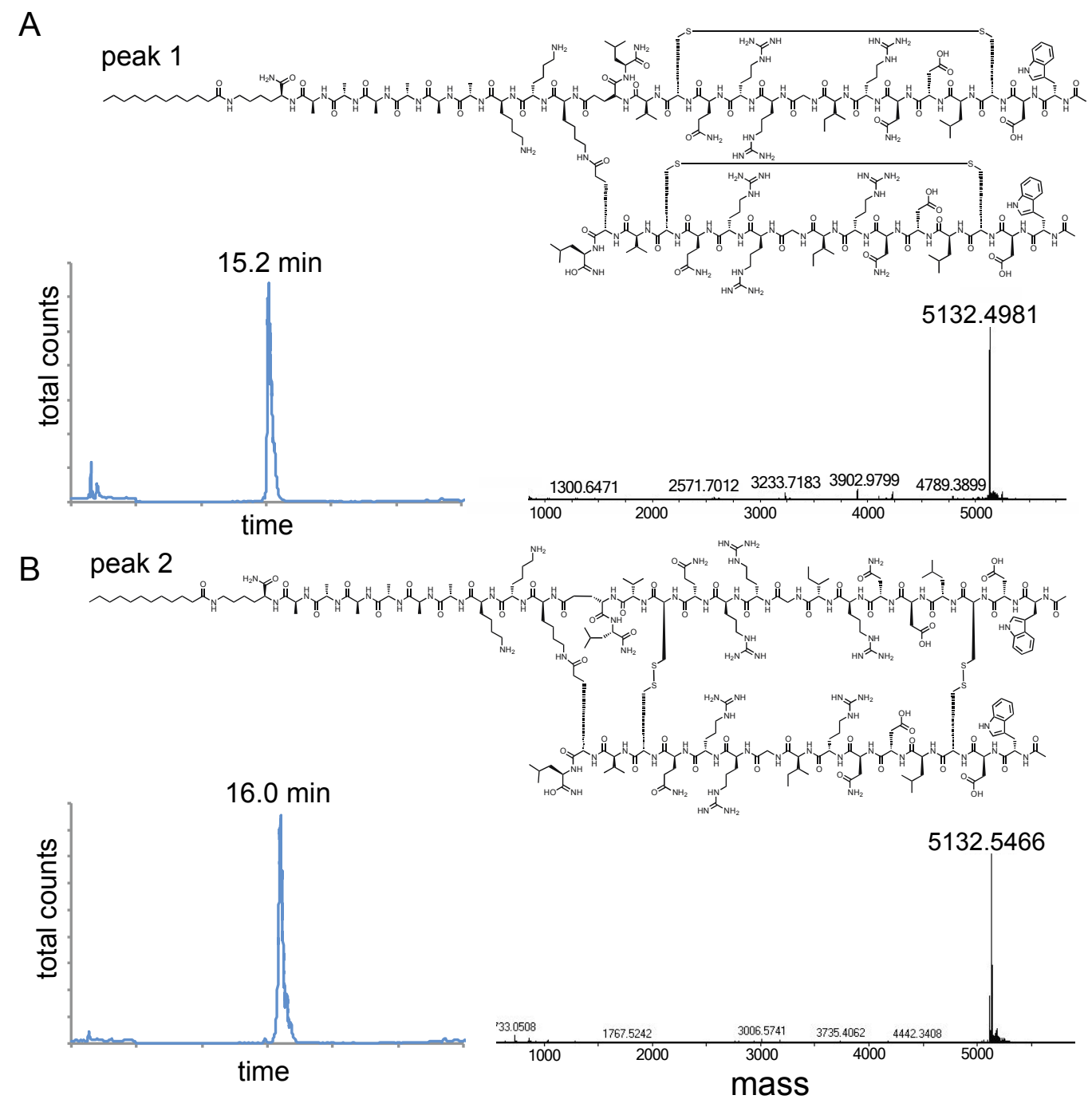

Figure S3: Characterization of the cyclized products of PA 1. Peaks 1 and 2 reference the HPLC trace in Figure 1B of the main text. (A) HPLC trace of the desired cyclized product, as demonstrated in supporting figure 4. (B) HPLC trace of the major side product of the cyclization reaction. Both peak 1 and peak 2 have the same mass as observed by ESI-MS. Similar HPLC traces for peptide 2, which lacks the self-assembly domain, were observed during the same processing procedure. 
A

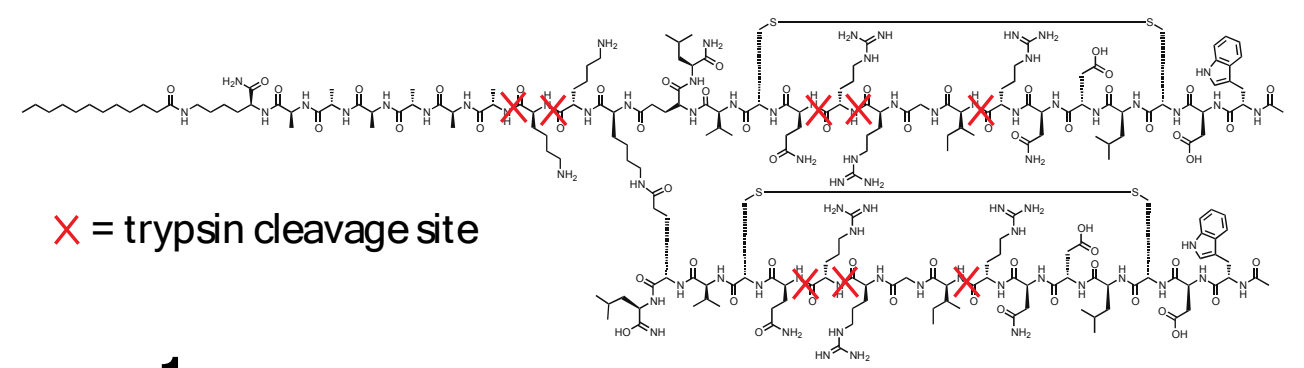

B
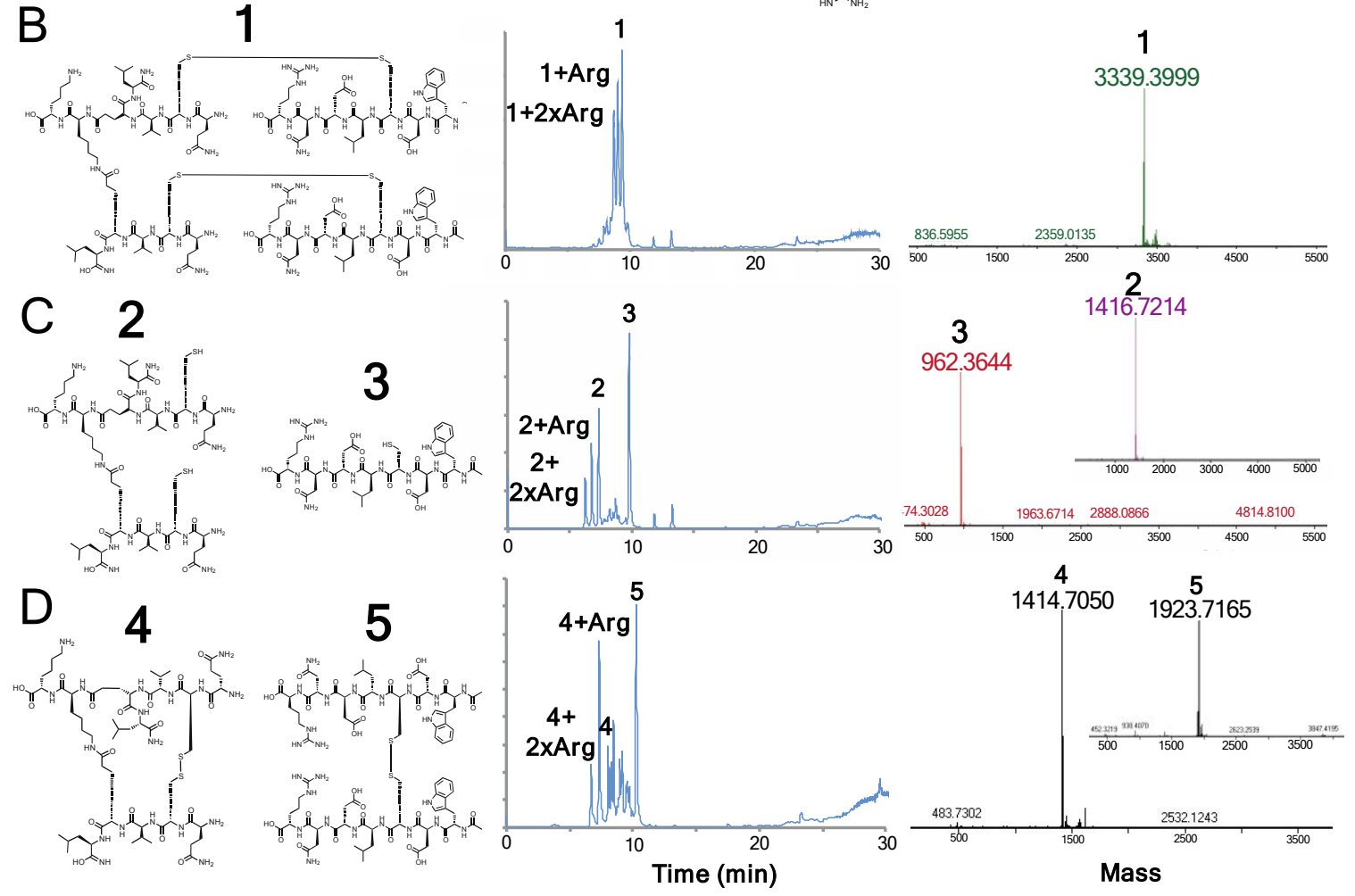

Figure S4: Determination of the cyclization reaction products by trypsin digestion. (A) Structure of PA 1 shows the sites where trypsin will cleave the peptide bond, at the carboxyl side of positively charged amino acids (lysine and arginine). The resulting degradation products differentiate the two PAs described in supporting figure 3, even though the intact PAs have the same mass. (B) Degradation product of the first eluted peak (Figure S3A) shows the expected degradation product for the correctly cyclized product by LC-MS. Compound $\mathbf{1}$ is shown with the corresponding HPLC and mass spec traces. 1+Arg represents compound $\mathbf{1}$ with an additional arginine from incomplete trypsin digestion. (C) After adding TCEP to the compound 1, the reduced form shows the expected mass from the products (compounds $\mathbf{2}$ and $\mathbf{3}$ ) with reduced thiols, showing that the cyclization is the result of disulfide formation. (D) The degradation product of peak 2 from Figure S3B shows the disulfides 
forming between the binding sequences instead of two cyclic structures, producing compounds $\mathbf{4}$ and 5. Similar to compound $\mathbf{1}$, incomplete digestion is observed with masses that correspond to additional arginine residues. Peaks with extra arginine residues were observed in both cases, possibly because the disulfide cyclization slows the reaction rate of the protease, or because the reaction was performed at the suboptimal pH 6.5 to prevent disulfide scrambling; however, these additional peaks did not interfere with our ability to determine the location of disulfide bonds.
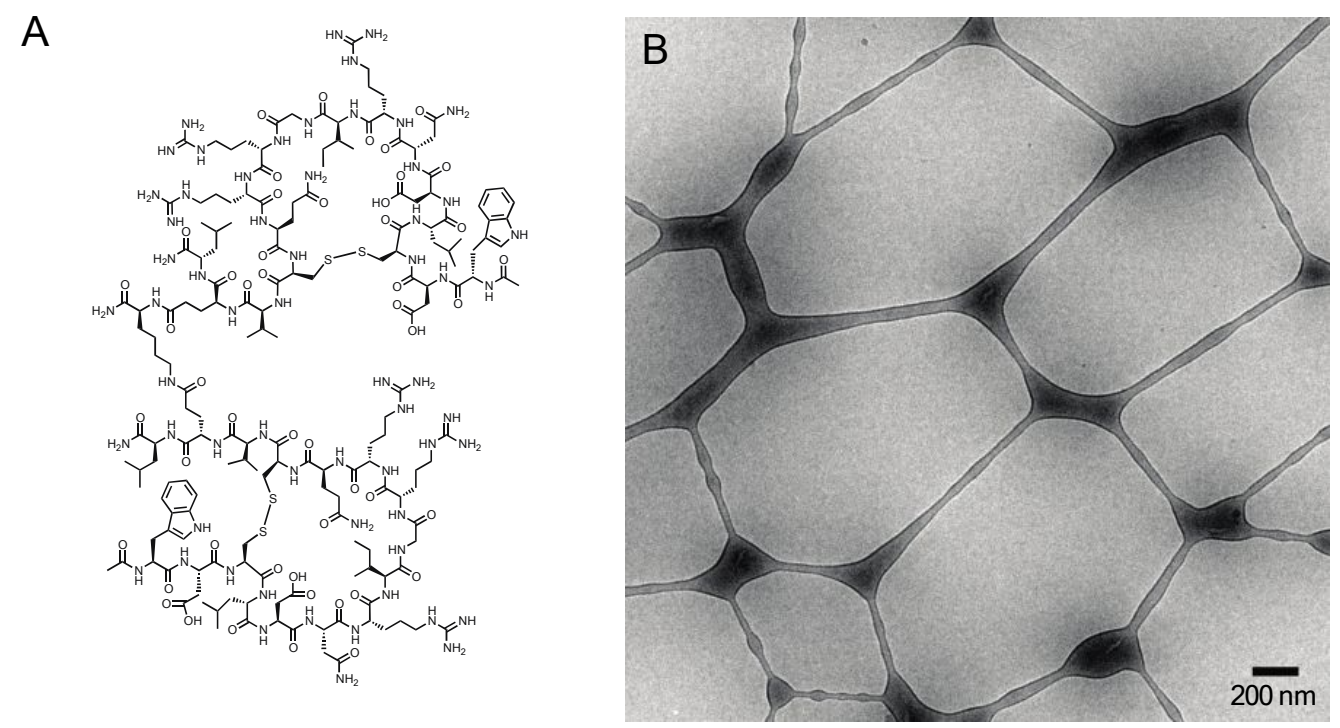

Figure S5: Characterization of peptide 2. (A) Chemical structure of peptide 2, which contains two binding sequence to DR5, but not the self-assembly domain. (B) Cryo-TEM of $100 \mu \mathrm{M}$ peptide in PBS did not show any nanostructures in solution. 
A

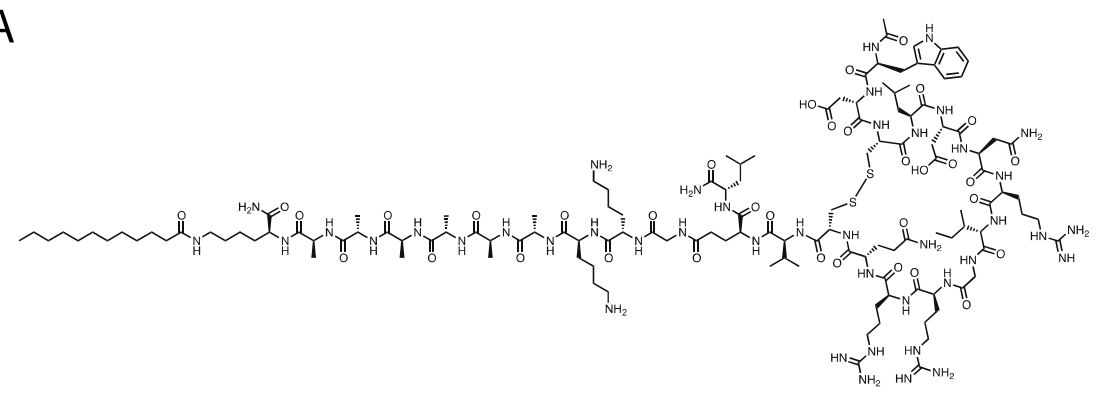

B

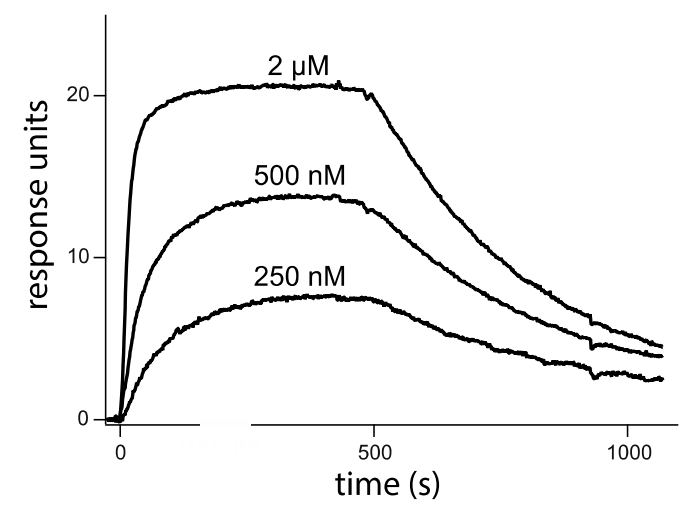

Figure S6: Characterization of PA 3. (A) Chemical structure of PA 3, which contains a self-assembly domain, but with a single DR5-binding sequence. (B) SPR of PA 3 showed binding at higher concentrations relative to the dimeric PA and peptide.

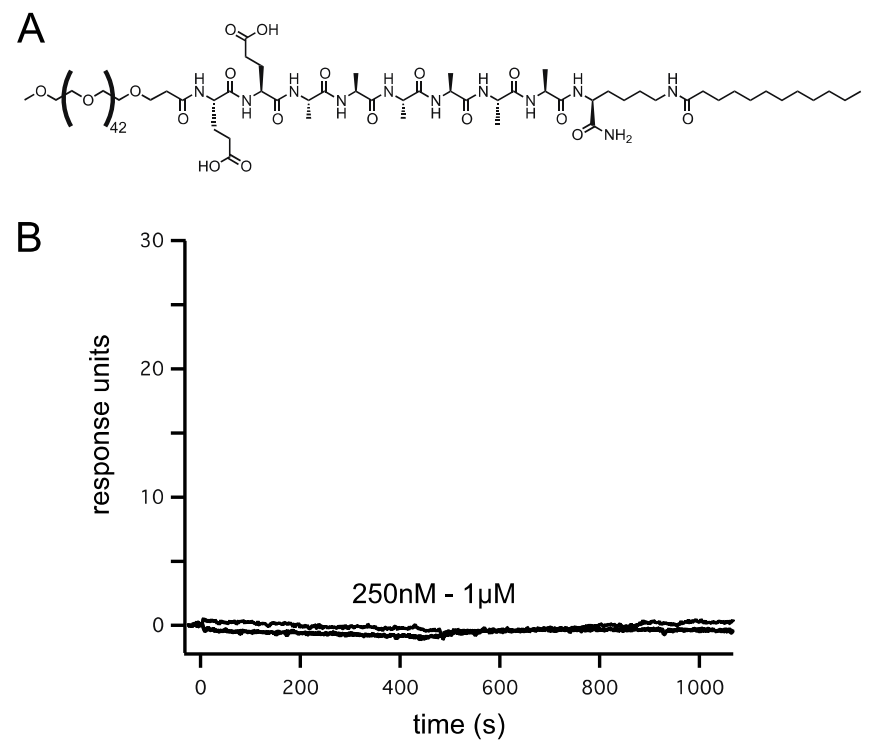

Figure S7: PEGylated PA (PA 4) and its interaction with DR5. (A) The chemical structure of PA 4 is shown, which has the same $\beta$-sheet domain as PA 1. (B SPR of PA 4 against DR5. 

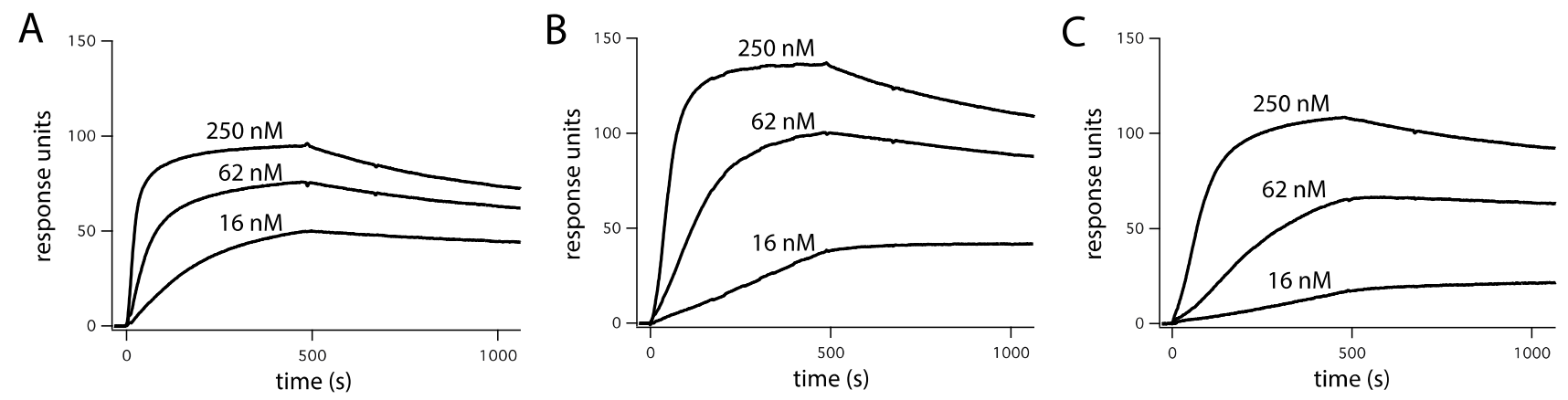

Figure S8. Surface plasmon resonance curves of peptide 2 (A), PA 1 (B), and coassembled PAs 1 and 4 (C) at three different concentrations. Concentrations in (C) indicate the concentrations of PA 4, not the total PA concentration.

A

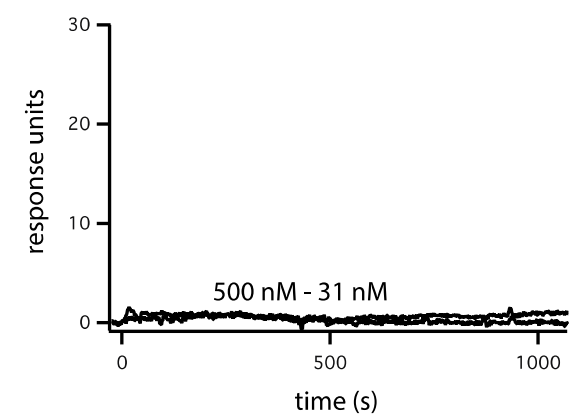

B

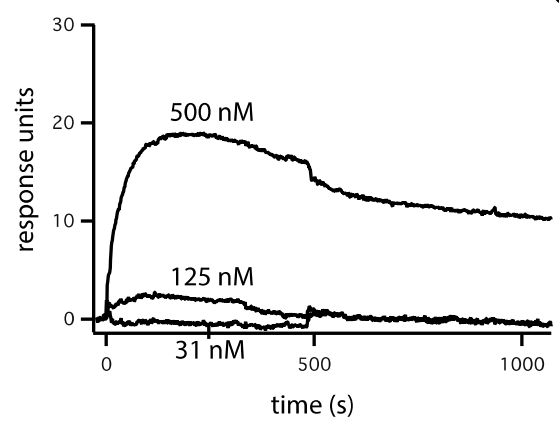

C

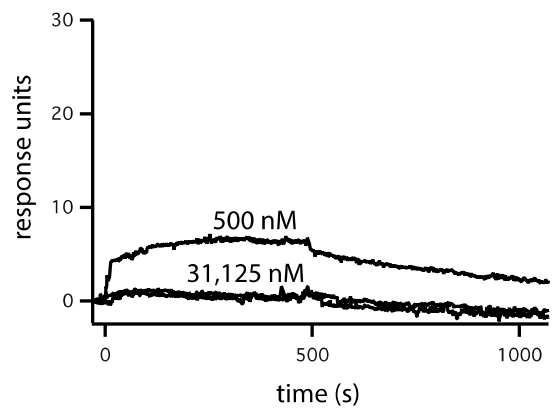

Figure S9: Surface plasmon resonance of PAs and peptides against DR4. (A) Peptide 2 did not interact with DR4 at concentrations up to $500 \mathrm{nM}$. (B) Some non-specific binding was observed for PA 1. (C) This non-specific binding decreased for the coassembled case of PAs 1 and 4. Concentrations in (C) represent the concentration of PA 4. 

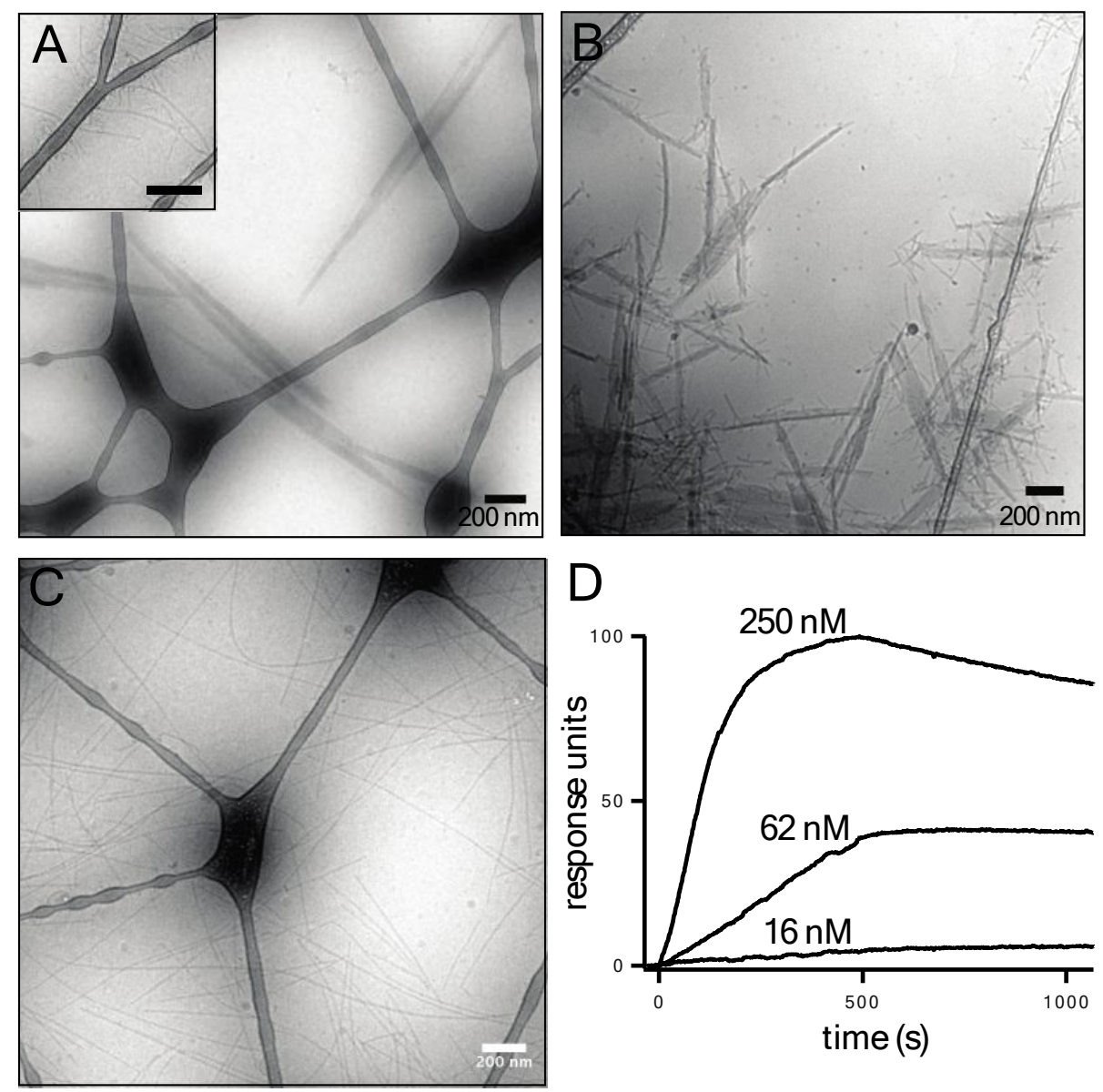

Figure S10: Cryo-TEM of PAs with encapsulated paclitaxel. (A) Cryo-TEM shows PAs 1 and 4 nanostructures with encapsulated paclitaxel at a concentration of $16 \mu \mathrm{M}$ PA 1 and $33 \mu \mathrm{M}$ PA 4 in PBS. Inset shows the existence of narrower nanostructures that are roughly $10 \mathrm{~nm}$ in width. (B) $50 \mu \mathrm{M}$ PA 4 with paclitaxel showed the same mix of aggregated structures and short fiber structures. (C) With the same PA concentrations as (A) and coassembled in the presence of DMSO but not paclitaxel, cylindrical structures were observed. (D) SPR of PAs $\mathbf{1}$ and $\mathbf{4}$ with paclitaxel shows comparable binding kinetics to coassembled PAs without paclitaxel. 


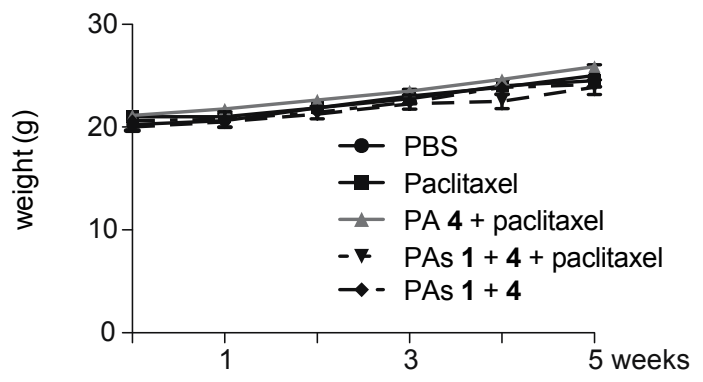

Figure S11: Mouse weights plotted as a function of time show that therapies have now affect relative to the saline control. 